Paper

\title{
Stochastic resonance induced by internal noise in a unidirectional network of excitable FitzHugh-Nagumo neurons
}

\author{
Kazuyoshi Ishimura ${ }^{1 a)}$, Alexandre Schmid ${ }^{2}$, Tetsuya Asai ${ }^{1}$, \\ and Masato Motomura ${ }^{1}$ \\ ${ }^{1}$ Graduate School of Information Science and Technology, Hokkaido University \\ Kita 14, Nishi 9, Kita-ku, Sapporo, Hokkaido, Japan \\ ${ }^{2}$ Microelectronic Systems Laboratory, École Polytechnique Fédérale de \\ Lausanne, CH-1015, Lausanne, Switzerland \\ a)ishimura@lalsie.ist.hokudai.ac.jp
}

Received August 20, 2015; Revised October 20, 2015; Published April 1, 2016

\begin{abstract}
Stochastic resonance (SR) is a phenomenon in which dynamic noise is effectively used to drive a system with subthreshold input signals. In the classical model of SR induced in a network, each constituting unit must be delivered noise from independent external sources. Recently, a new model of SR has been proposed, where internal noise is exploited as the solution to avoid the burden of generating independent external noise for each unit. In this study, we employ a network of FitzHugh-Nagumo neurons as a candidate of the new model of SR system using internal noise. The network is formed as a circular system where all connections between neurons strictly consist of self-connections or connections propagating into a unique direction. Hence, each neuron receives stimuli from its four predecessors within the circular arrangement, from its own output, as well as from a unique input node. An input signal of an amplitude that is smaller than the threshold of individual neurons is provided to the network. Owing to a process of SR that occurs within the network and that is sustained by internally generated noise, the subthreshold signal is detected and amplified and delivered to the network output node. The frequency characteristics of the network in terms of its operational bandwidth is established.
\end{abstract}

Key Words: stochastic resonance, unidirectional network, FitzHugh-Nagumo neuron

\section{Introduction}

Noise is present in all natural phenomena, and influences or perturbs them depending on its nature and intensity. Some biological systems have been observed to process information based on external or thermal noise, e.g., [1,2]. Engineered systems and specifically sensory systems, information storage, processing and transmission systems and microelectronic systems also suffer from the presence of various types of noise that limit their performances in terms of resolution, bandwidth, access time, 


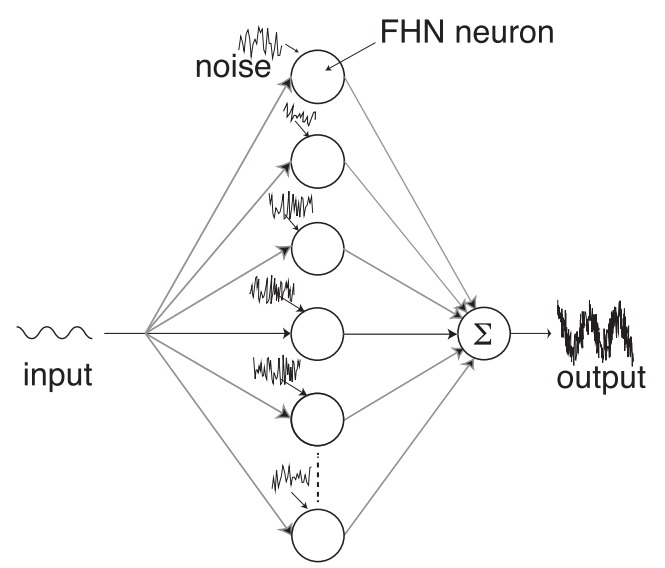

Fig. 1. Collins summing network topology [11].

for example. Noise has traditionally been considered an adverse phenomena that should be cancelled using complex fabrication technology, circuit and systems, or signal processing techniques, e.g., [3, 4]. The concept of taking benefit of the inherent existence of noise rather than investing much energy to combat it has recently been devoted increasing attention, particularly from the circuit and systems research communities, [5-9]. Improving the randomness of on-chip generated binary sequences used in various applications such as cryptography, local perturbation-based gradient descent algorithms that process optimization learning in artificial neural networks and can extract themselves out of a local minima of the energy function that must be minimized are such examples, e.g., [10]. Stochastic resonance (SR) has been identified as a phenomena in which a signal with subthreshold amplitude may be effectively detected under specific conditions of the environmental noise. SR is observed as an increase of the output signal-to-noise ratio (SNR) upon the presence or injection of a specific amount of noise. Hence, SNR is non-monotonic with respect to the amount of noise, and SR occurs at the maxima of the curve where a subthreshold signal becomes detectable by the system. SR has been used in Collins neural network model, exploiting external noise to support the recovery of an aperiodic subthreshold input signal by a network of FitzHugh-Nagumo (FHN) neurons, [11]. In this network, Fig. 1, every neuron's inputs consist of the network input as well as external noise, while the outputs converge to a summing node. Subthreshold and undetectable signals can be recovered at the network output in a noisy envelope.

On the other hand, another type of SR which is called chaotic resonance (CR) has been investigated using chaotic systems. CR can detect a subthreshold input signal by utilizing internal chaotic fluctuations which are induced by chaotic dynamics instead of some external noise source. Furthermore, CR has been observed in the electrical activity of some regions of the brain, where internal noise is used to support the emergence of SR, e.g., $[12,13]$. CR has been used in various microelectronic circuits, for example exploiting internal chaotic fluctuations in $[14,15]$.

Exploiting internally generated noise appears a relevant method that has been promoted by nature in the evolution of the brain. In engineered systems such as microelectronic circuits, eluding issues related to generating and controlling external noise appears an advantage. This study exploits a new type of SR which utilizes internal fluctuations that are induced by the network operation as intrinsic chaotic fluctuations [16-19]. This study presents a circular network of FHN neurons which take advantage of weighted internal signals that are propagated in a unidirectional manner as noise, in order to detect a subthreshold input. Section 2 presents the network fundamental expressions and topology. The major characteristics of the network setup and regime operation are defined in Section 3. Section 4 presents a frequency analysis of the network and discusses its optimal operational frequency range.

\section{Unidirectional circular network of FHN neurons}

A circular network of FHN neurons is formed such that each neuron receives the external input signal stimuli, as well as the weighted contributions of its neighbors, as shown in Fig. 2. Each neuron's output 


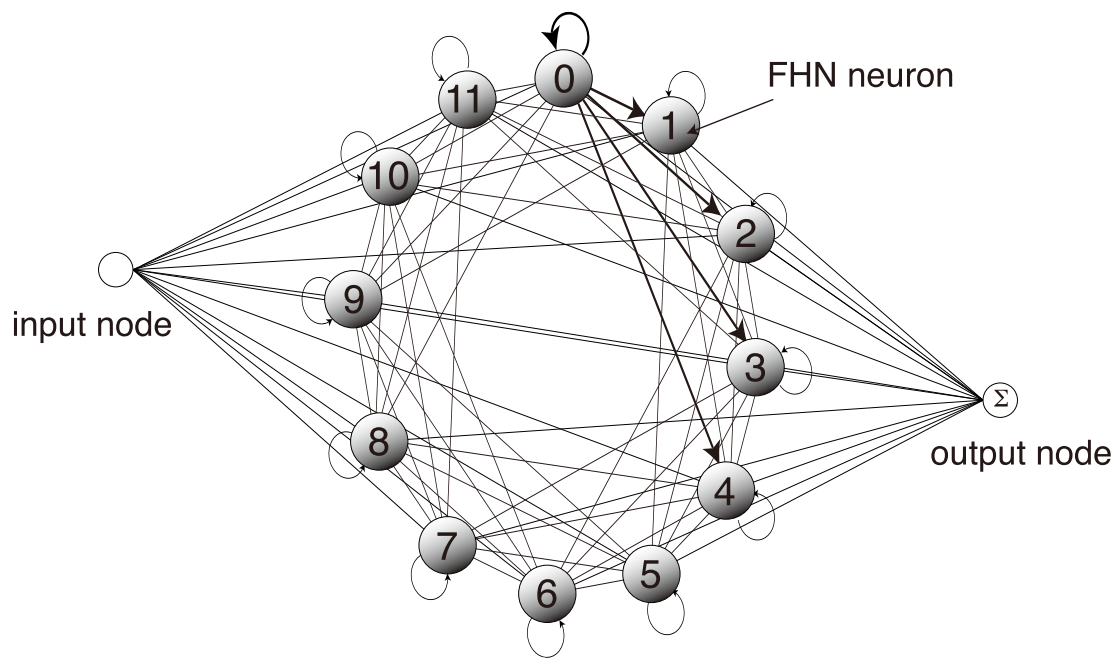

Fig. 2. Proposed unidirectional network of FHN neurons showing all unidirectional weighted connections.

is connected to a summing node which delivers the circuit output. Boundary conditions stipulate the continuous connectivity, i.e., the last neurons of a physical arrangement connect to the first neurons, following the weighted connectivity pattern that homogeneously applies to the circuit.

The dynamics of a FHN neuron is given by

$$
\begin{aligned}
& \frac{d u}{d t}=u(1-u)(u-a)-v+I(t), \\
& \frac{d v}{d t}=\epsilon(b u-v)
\end{aligned}
$$

where $t$ is the time, $u$ the membrane potential, $v$ the recovery variable, $\epsilon$ the time constant, $a$ and $b$ the system parameters, and $I(t)$ the input signal. In this study, we define $u$ as the output signal in a FHN neuron.

Here we assume that analog weighted signals originating from closely-located neighbors are able to act as noise, and the resulting dynamics that we propose is

$$
\begin{aligned}
& \frac{d u_{i}}{d t}=u_{i}\left(1-u_{i}\right)\left(u_{i}-a\right)-v_{i}+w I(t)+D \xi_{i}, \quad(i=1,2, \cdots, N) \\
& \frac{d v_{i}}{d t}=\epsilon\left(b u_{i}-v_{i}\right)
\end{aligned}
$$

where $N$ is total number of neurons, $i$ the neuron index, $w$ the strength of the input signal, $D$ the noise amplitude factor, and $\xi_{i}$ the noise input. In the case of bidirectional coupling among the neurons, $\xi_{i}^{B}$ can be represented by

$$
\xi_{i}^{B}=\frac{1}{\alpha_{0}+\sum_{j=1}^{n} \alpha_{i-j}+\alpha_{i+j}}\left(\alpha_{i} u_{i}+\sum_{j=1}^{n} \alpha_{i-j} u_{i-j}+\alpha_{i+j} u_{i+j}\right)
$$

where $n$ is the number of connecting neighbors $(n>1)$, and $\alpha$ the connection coefficient. $D$ enables adjusting the total noise level within the network, whereas $\alpha$ enables individually adjusting an input signal level originating from a neighbor neuron as a noise source.

Assuming that each neuron exhibits excitable properties [20], excitable waves propagate in both directions, which results in potential wave collisions that would suppress the sustained wave propagation. In order to guarantee the sustained wave propagation, we propose unidirectional coupling as 


\begin{tabular}{|c|c|c|c|}
\hline $\begin{array}{l}\text { number of } \\
\text { connecting neighbors } \\
n \\
0\end{array}$ & $\begin{array}{r}M \\
0\end{array}$ & $\alpha_{i-5} \alpha_{i-4} \quad \alpha_{i-3} \quad \alpha_{i-2} \quad \alpha_{i-1} \alpha_{i} \quad \alpha_{i+1} \quad \alpha_{i+2} \alpha_{i+3} \alpha_{i+4} \alpha_{i+5}$ & $\begin{array}{c}S\left(2^{M}\right) \\
1\end{array}$ \\
\hline & 1 & 11 & 2 \\
\hline 1 & 2 & 2 & 4 \\
\hline & 3 & 133 & 8 \\
\hline 2 & 4 & $\begin{array}{llll}4 & 6 & 4 & 1\end{array}$ & 16 \\
\hline 3 & $\frac{5}{6}$ & ${ }_{1}^{1}{ }^{1}{ }^{5}{ }_{15}{ }^{10}{ }_{20} 10_{15}{ }^{5}{ }_{6}^{1}$ & $\frac{32}{64}$ \\
\hline & 7 & $\begin{array}{llllllll}1 & 7 & 21 & 35 & 35 & 21 & 7 & 1\end{array}$ & 128 \\
\hline 4 & $\begin{array}{l}8 \\
9\end{array}$ & $\begin{array}{ccccccccccccc} & 1 & & 8 & 28 & 56 & 70 & 56 & 28 & 8 & & & \\
1 & 9 & 36 & 84 & 126 & 126 & 84 & 36 & 9 & & 1\end{array}$ & $\begin{array}{l}256 \\
512\end{array}$ \\
\hline 5 & 10 & $\begin{array}{lllllllllll}1 & 10 & 45 & 120 & 210 & 252 & 210 & 120 & 45 & 10 & 1\end{array}$ & 1024 \\
\hline
\end{tabular}

Fig. 3. Pascal's triangle representing discretized bidirectional coefficients.

$$
\xi_{i}^{U}=\frac{1}{\sum_{j=0}^{n} \alpha_{i-j}}\left(\alpha_{i} u_{i}+\sum_{j=1}^{n} \alpha_{i-j} u_{i-j}\right)
$$

Here we assume that the wave propagation is caused by a diffusion process, which results in having the Gaussian impulse response. Hence, the $\alpha$ values are selected as the coefficient of the Gaussian function which implements a non-linear function of the coupling factor with respect to the distance between the neurons. In order to discretize the Gaussian function, we used Pascal's triangle, as shown in Fig. 3, where $M$ represents the kernel index, and $S$ represents the sum of all coefficients, $n(\equiv\lfloor M / 2\rfloor)$ represents the number of connecting neighbors from the center $(i)$. For example, when $n=4$, we obtain

$$
\left.\xi_{i}^{U}\right|_{n=4}=\frac{70 u_{i}+56 u_{i-1}+28 u_{i-2}+8 u_{i-3}+u_{i-4}}{163},
$$

which consists of weight connections following a rule based on the distance between the recipient $\left(u_{i}\right)$ and sources $\left(u_{i-1}, u_{i-2}, u_{i-3}\right.$, and $\left.u_{i-4}\right)$; the rule homogeneously applies in the network. The weighted contributions of the neighboring neurons consist of an analog signal that can be considered an internal noise; however, the behavior of the network is deterministic, and is predicted by the equations governing its dynamics.

\section{Operation of the unidirectional system}

In order to study several of the fundamental characteristics of the operation of the network, an external sinusoidal input $I(t)$ which has suprathreshold amplitude of 0.075 as a bootstrap input signal for generating internal noise, and has subthreshold amplitude of 0.05 later, and has an input frequency of $10^{-4} \mathrm{~Hz}$ is applied in a circular network consisting of 500 neurons. At first, the system parameters of the neurons are set to $a=0.1$ with a variation of $5 \%, b=0.24$ with a variation of $1 \%$. Using these parameters, the internal states of each neuron converge to 0 without a bootstrap phase and an input signal. Neuron firings may follow different individual dynamics, depending on the initial conditions, as well as the cumulated amplitude of external and internal stimuli. This sustained level of activity impacts on the stimulation of forward-neighboring neurons, and thus on the wave propagation. Henceforth, this metrics is used in the raster plot analysis consisting of the timespan between an upwards and the downwards relaxation crossings of a threshold in the amplitude, rather than the time of the spiking usually defined from a unique threshold crossing condition. The threshold is selected at 0.6 , i.e., slightly lower than the value observed on the constant plateau. Consequently, the raster plots bear information related to the timing and timespan of the neuron firings as well as their impact on the generation of travelling waves.

The collection of neurons that simultaneously fire when their internal states are higher than a threshold value equal to 0.6 within one determined time-window, is herein named a bundle and is drawn using red lines in Fig. 4, and is observed as the number of firing neurons within the segment marked "a" which is extracted from the vertical axis as approximately 10 neurons. This number 


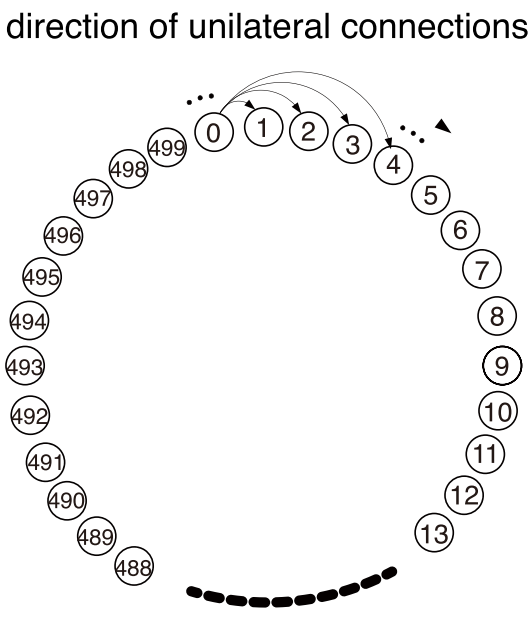

(a)

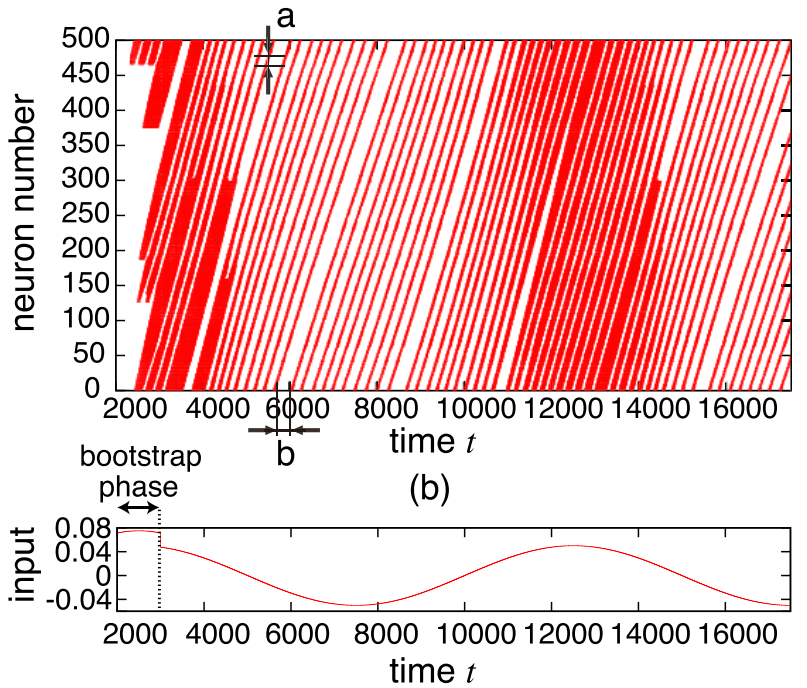

(c)

Fig. 4. Generation of traveling waves. (a) Graphical model of the circular network, (b) raster plot, (c) input signal which has a bootstrap input signal $I(t)=0.075 \sin (2 \pi f t)$ when $t<3,000$ and a subthreshold input signal $I(t)=0.05 \sin (2 \pi f t)$ when $t \geq 3,000$. The concomitant firing event of several consecutive neighboring neurons, clearly showing the bundles forming traveling waves. The parameters of the neurons are set to $a=0.1$ with a variation of $5 \%, b=0.24$ with a variation of $1 \%, \epsilon=0.01$ with a variation of $3 \%, w=0.045$ with a variation of $1.8 \%$, and the noise amplitude factor $(D)$ which weights all noise as well as self-feedback connections is set to $D=0.057$.

typically varies in time, which exhibits the stochastic nature of the process and confirms the interpretation of the internal stimuli as noise. In Fig. 4, a chain of firing neurons is observed to induce firing in the downstream-located neurons that exploit prior firings as internal noise. The number of neurons participating in a bundle is a function of the amplitude of the stimuli, the number of forward connections and their strengths, the initial random conditions as well as the instantaneous status of variability-subjected parameters of the FHN neurons. An analytical expression of the number of neurons in a bundle can be determined by iteratively applying the noisy FHN neuron expression in (1) and (2), including variability and random initializations. Hence, a rigorous algorithmic description of this process appears impractical in terms of its applicability.

Every neuron in the network is consecutively stimulated to fire, immediately following its predecessor in the chain forming a chain-reaction. Hence, a wave propagates which circulates around the network. Nevertheless, the wave that travels throughout the network consists of the consecutive firing of all neurons which participate in consecutive bundles. In addition to internal parameters primarily involving the refractory period of each neuron and the time-constant of the low-pass filter created by the neuron interconnectivity, the propagation speed has a strong dependence on the frequency of the stimuli.

Each neuron in a bundle fires a certain number of times, and obeying its own firing frequency that is dictated by its internal refractory period. This time-window of consecutive firings is named period of activity. In the case of a periodic input, the period of activity which corresponds to the highamplitude period of the stimuli is followed by a period of low activity or inactivity, which corresponds to the low-amplitude phase of the stimuli. The inactivity period of neuron No. 0 is observed as the time interval marked "b" in Fig. 4. Eventually, a new bundle is stimulated which starts a new wave propagating through the network. At any time, several waves propagate through the network.

New bundles form in the network during the high-amplitude phase of the stimuli, mostly. Figure 4 shows the generation of several bundles during a bootstrapping phase by $t=3,000$, and exclusively during which a stimuli of extremely high amplitude is applied, at startup of the system for generating internal noise. Following their generation, waves propagate and upon reaching neuron No. 499 continue at neuron No. 0, hereby obeying cyclic boundary conditions. Variability of individual neuron's 
parameters dictates a degree of randomness in the generation of propagating waves, which may also enable the generation of a new bundle during the low-amplitude phase of the stimuli, in rare cases. Along time, the density of waves propagating in the network, defined as the number of bundles that exist at a certain time, varies.

The wave propagation through the network is sustained by internal noise, and the propagation also proceeds in absence of external stimuli. This phenomena is absolutely crucial to the correct operation of the network in subthreshold stimuli, since the waves represent permanent internal noise that is required to support the SR process that enables extracting subthreshold signals by creating a peak of SNR at some appropriate noise level. The propagation speed of a wave through the network is governed by the dynamics of individual FHN neurons that consecutively stimulate their forward neighbors in a chained reaction. The circular configuration of the network promotes self-stimulation into a stationary state, under conditions that enable the stability of the system. The general topology of the circular network resembles a ring oscillator's, and an approach which is inspired by the extensive study carried over ring oscillators can be applied to express the conditions of self-oscillation of the network, or expressed differently, the necessary condition that allow several waves to sustain themselves inside the circular network.

$\mathrm{SR}$ is evidenced from a peaking correlation value that is obtained at a certain level of noise within the network. The correlation value $(C)$ is given by

$$
\begin{aligned}
C & =\frac{\langle I(t) \cdot O(t)\rangle-\langle I(t)\rangle\langle O(t)\rangle}{\sqrt{\left\langle I(t)^{2}\right\rangle-\langle I(t)\rangle^{2}} \sqrt{\left\langle O(t)^{2}\right\rangle-\langle O(t)\rangle^{2}}}, \\
\langle X(t)\rangle & \equiv \frac{1}{T} \int_{t-T}^{t} X(t) d t
\end{aligned}
$$

where $I(t)$ represents the common input, $O(t)$ the summation of the outputs of FHN neurons, $T$ the time window of correlation calculation.

In practical terms, the correlation value ranges over $[-1,1]$; though any value in the range $[-0.5,0.5]$ indicates the absence of correlation, and the calculation method does not allow any additional meaningful interpretation; values close to -1 or 1 are indicative of correlation.

As shown in Fig. 5(a), the amplitude of noise become large when the noise strength $D$ is increased. Therefore, the system parameter $D$ contributes to the characteristics of internal noise. Figure $5(\mathrm{~b})$ shows that the weighted sum $\left(\xi_{0}\right)$ of its own predominant firing (neuron No.0) and four predecessors firing contributions behave as internal noise which has power at frequencies higher than the input signal. The analysis of the relationship between the correlation value and noise strength $D$ presented in Fig. 5(c) enables observing a clear SR behavior in the form of a maximum (peak) of the correlation value that is obtained by adjusting an appropriate amount of noise as shown in $[19,21]$. When the noise amplitude is increased from a low amplitude value, the correlation value is known to increase in any network. When the number of FHN neurons is small $(N=50,100,200)$, the correlation value decreases after reaching a peak value. As shown in Fig. 6, the noise generated in a configuration consisting of a single coupling neuron is not sufficient to sustain propagating waves. The correlation value is observed to increase with a higher number of coupling neurons, showing over $90 \%$ of correlation value if $n \geq 4$, a saturation level, and sharply decreasing at a number of couplings higher than 9 at $90 \%$ of correlation value, due to an over-averaging effect through the weighted summation of activities of a neuron's neighbors as noise.

\section{Frequency response of the unidirectional FHN neural network}

The frequency performance analysis of the network pertains to the capacity of the network to sustain propagating waves in the presence of an input signal of high frequency. The frequency bandwidth of individual FHN neuron has an influence on the global network behavior; its study has been carried out earlier, and is not repeated in this work. Different operation regimes and an optimum window of input frequency operation are explained in the following, and the trade-offs involved in the improvement of the window of correct operation are discussed. 


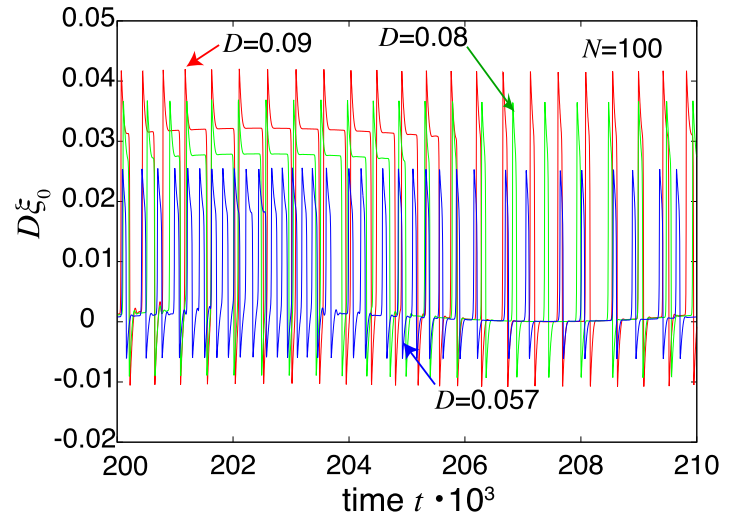

(a)

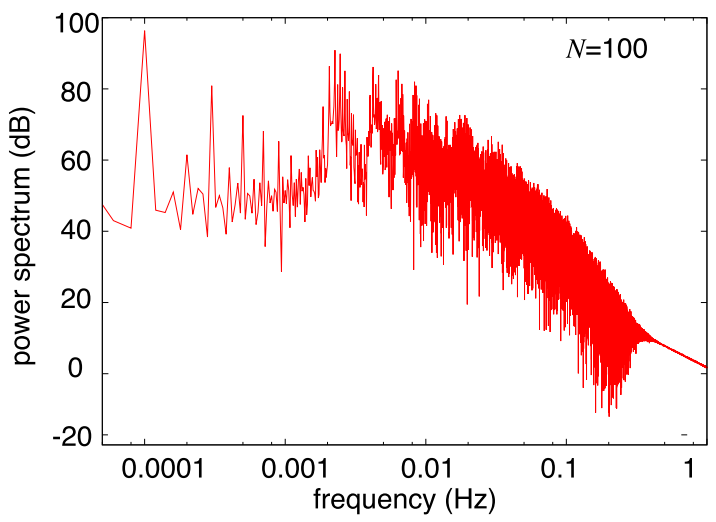

(b)

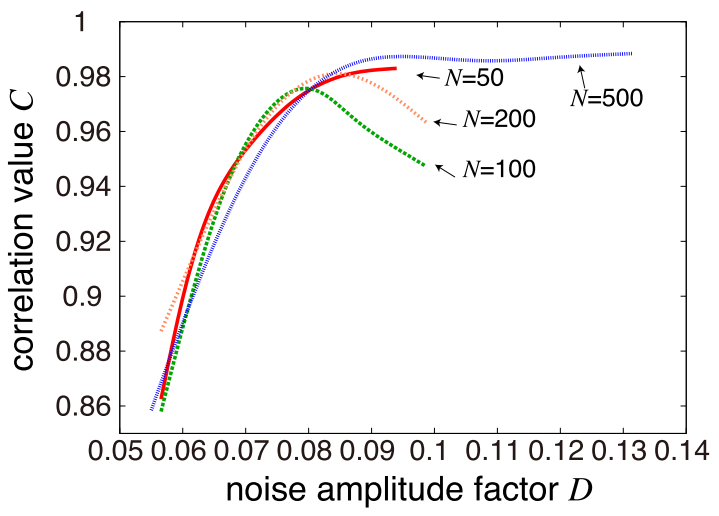

(c)

Fig. 5. (a) Noise term $D \xi_{0}$ which is applied to neuron No. 0 and consists of weighted summation of activities from itself and four predecessor neurons $(N=100)$. (b) Power spectrum of the noise term $D \xi_{0}$ which has power in a wider bandwidth than the sinusoidal input signal $(N=100$ and $D=0.09)$. (c) Correlation value with respect to the noise amplitude for different numbers of neurons in the network $(N=50,100,200,500)$. The following conditions are assumed, $a=0.1$ with a variation of $5 \%, b=0.24$ with a variation of $1 \%$, $\epsilon=0.01$ with a variation of $3 \%, w=0.045$ with a variation of $1.8 \%, I(t)=$ $0.075 \sin (2 \pi f t)$ when $t<3,000$ and $I(t)=0.05 \sin (2 \pi f t)$ when $t \geq 3,000$, and input frequency $f=10^{-4} \mathrm{~Hz} . T$ is set at 250,000 .

The frequency behavior study of electrical systems is traditionally based on the amplitude response of the system to a sinusoidal input of a frequency varying over a specified bandwidth. The amplitude is selected as an optimal metrics that is suitable to identify the transfer function of a single-output system. Though, the unidirectional circular network of FHN neurons presented in this research formally has one single output, the signal observed from this output is a linear combination of multiple signals generated by non-linear oscillators. Furthermore, the system performance is not assessed in terms of the system amplification, but in terms of the capacity of the system to extract a subthreshold input signal. Expressed differently, though amplification occurs and is a necessary condition to the operation of the system, its performance can not be defined in terms of pure amplification that would indifferently affect input signal as well as external and internal noise, but is correctly identified as the correlation value previously defined in (3).

In Fig. 7, four ranges of interpretation of the network response are identified, which are discussed in detail in the following subsections, namely:

- FR1: plateau of the spectral response where correct network behavior is observed;

- FR2: first roll-off of the spectral response where correct behavior is still observed, and hence operation within the part of this region over the correlation value of 0.5 is acceptable;

- FR3: second roll-off and bottom plateau of the spectral response where incorrect behavior is 


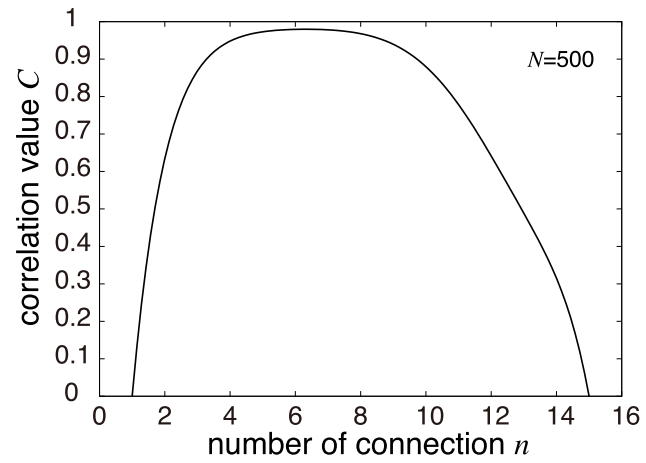

Fig. 6. Correlation value with respect to the number of unilateral coupling connections when $D=0.08$. The following conditions are assumed, $a=0.1$ with a variation of $5 \%, b=0.24$ with a variation of $1 \%, \epsilon=0.01$ with a variation of $3 \%, w=0.045$ with a variation of $1.8 \%, I(t)=0.075 \sin (2 \pi f t)$ when $t<3,000$ and $I(t)=0.05 \sin (2 \pi f t)$ when $t \geq 3,000$, and input frequency $f=10^{-4} \mathrm{~Hz}$. $T$ is set at 250,000 .

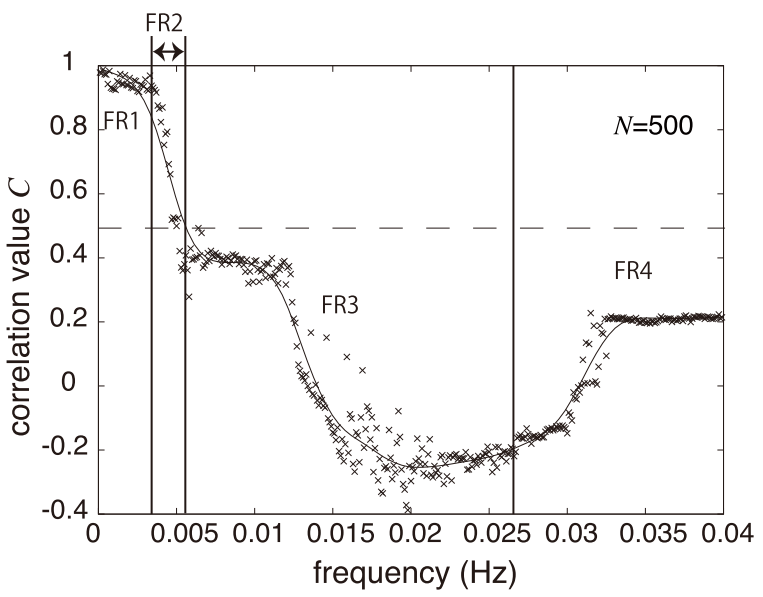

Fig. 7. Frequency response of the network. The frequency response of the system is presented, for a circuit configuration defined as follows, $a=0.1$ with a variation of $5 \%, b=0.24$ with a variation of $1 \%, \epsilon=0.01$ with a variation of $3 \%$, $w=0.045$ with a variation of $1.8 \%, N=500$, the noise amplitude factor $D=$ 0.057. Input signal which has a bootstrap input signal $I(t)=0.075 \sin (2 \pi f t)$ when $t<3,000$ and a subthreshold input signal $I(t)=0.05 \sin (2 \pi f t)$ when $t \geq 3,000$.

observed;

- FR4: uptake of the spectral response in the high frequency range, where incorrect behavior is observed.

\section{Frequency ranges FR1 and FR2}

The frequency response of the network shows a plateau at low frequency. In this domain of operation, the network operates properly, and according to its expected behavior, i.e., SR occurs and enables the detection of a subthreshold input signal. Within the first part of the roll-off of the frequency spectrum, correct operation of the network is preserved. The time-domain operation of the network stimulated with a subthreshold input at a frequency of $10^{-4} \mathrm{~Hz}$, and the time-domain operation is shown in Fig. 8. The red line represents a sinusoidal input which has a bootstrap behavior when $t<3,000$; the green spiking line represents the time course of $u_{0}$, and the blue line represents the network output.

Optimally, the network should be operated within the region of the plateau, that guarantees the proper conditions supporting the SR process. We consider the correlation value as the source of identification parameter as well as the fact that the part of zone FR2 is acceptable over the correlation of the value 0.5 as shown in Fig. 9, at a frequency of the input stimuli of $0.005 \mathrm{~Hz}$. 


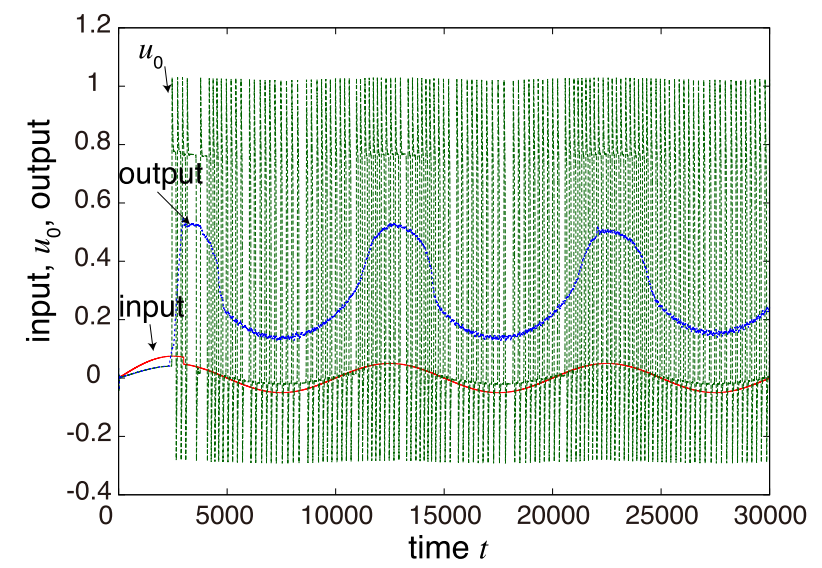

Fig. 8. Time-domain operation of the network that correctly operates in FR1.

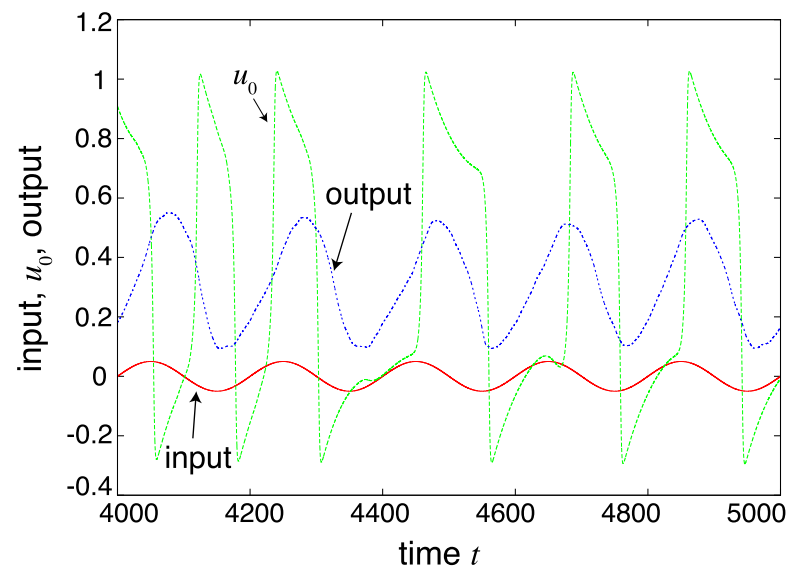

Fig. 9. Time-domain operation of the network that operates acceptably in FR2.

\section{Frequency range FR3}

The network should not be operated outside of its frequency range of correct operation, such as frequency ranges FR3 and FR4. In addition, the calculation method applied to processing the correlation value does not yield result in the range of $[-0.5,0.5]$ that may adequately be interpreted. Figure 10 shows the time-domain operation of the circuit in FR3, at a frequency of the input stimuli of $0.02 \mathrm{~Hz}$. In frequency range FR3, the network output does not follow the input. The signal is integrated by the neurons due to the excessive input frequency, and a neuron output is observed as switching at a lower frequency than the input.

Two interesting features are observed in the time-domain curve. First, the output integrates a number of input cycles prior to switching. In a large network, the number of integrators that contribute to the integration is large. Eventually, the switching time can not be predicted with certainty, and the switching period time is chaotic. Second, the subthreshold input signal is observed as a contributor to the output signal. Direct neuronal input-to-output coupling is the cause of this phenomena.

\section{Frequency range FR4}

Frequency range FR4 as FR3 represents a frequency range that is not adapted to the proper operation of the network. Figure 11 shows the time-domain operation of the circuit in FR4 at a frequency of the input stimuli of $0.035 \mathrm{~Hz}$. The difficulty of neurons to following the subthreshold stimuli is more pronounced than in FR3. Nevertheless all parts of the curve located within the limits of the coefficient factor of $[-0.5,0.5]$ should be carefully interpreted, and the exact curve is not relevant.

The correct operation of the network requires a bandwidth-limited input signal following the FR1 and FR2 criteria explained above. A method that enables increasing the operation bandwidth is discussed in the following.

Parameter $\epsilon$ is a determinant parameter in the dynamics of an individual FHN neuron which has a 


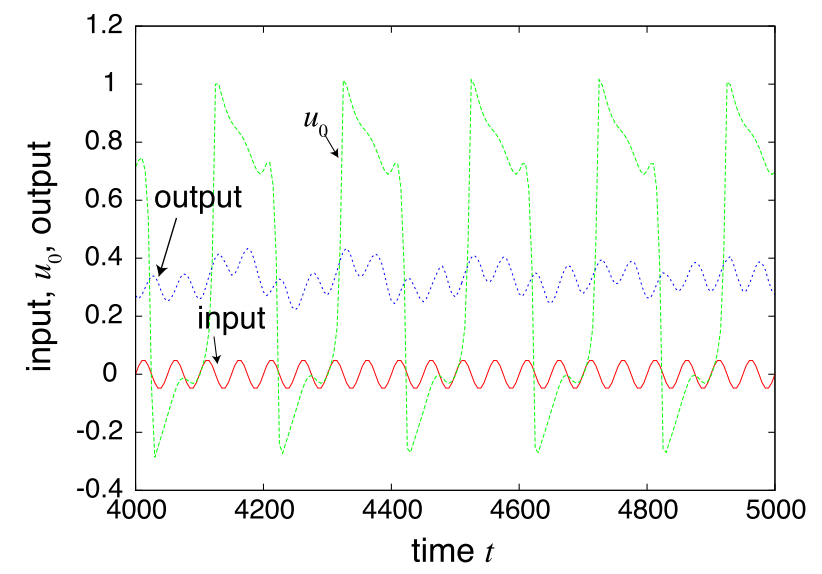

Fig. 10. Time-domain operation of the network that operates in frequency range FR3.

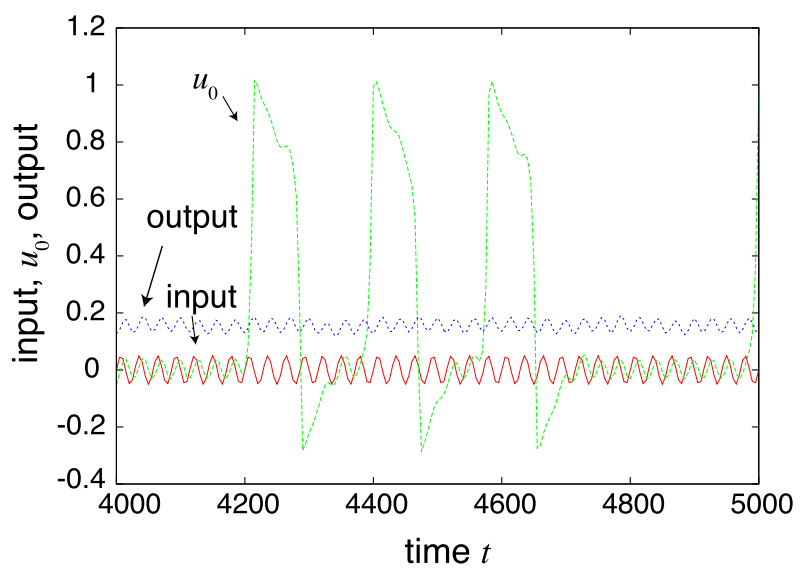

Fig. 11. Time-domain operation of the network that operates in frequency range FR4.

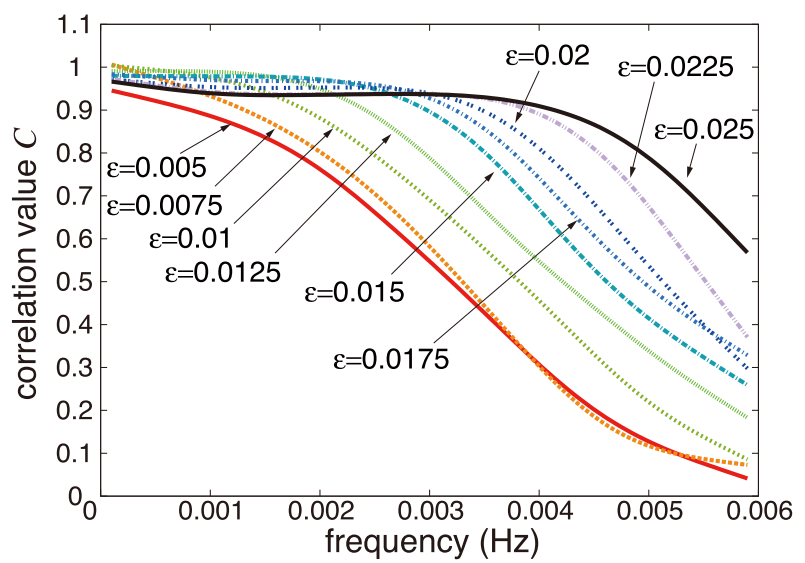

Fig. 12. Correlation values with respect to frequency and time constant $\epsilon$.

significant influence over recovery variable $v$, following expression (2). In physical terms, $\epsilon$ represents a time constant, and as such impacts the frequency response of individual neurons as well as the entire network. Figure 12 shows the frequency response of the circuit in terms of its coefficient factor. Nine values of $\epsilon$ are selected, each corresponding to a different response. A different extension of the plateau that determines FR1 is observed for increasing values of $\epsilon$. The curve shows an increase of the plateau of correct operation for increasing values of $\epsilon$, until saturation is reached. 


\section{Conclusion}

We proposed a nonlinear dynamical system exhibiting stochastic resonance (SR) induced by the system's internal activities (noise) as the solution to avoid the burden of generating independent noise for each unit so as to induce SR in the traditional manner. The system consists of a 1-D array of excitable FitzHugh-Nagumo neurons with cyclic boundary condition, where the action potential propagate on the 1-D space as excitable waves. Each neuron accepts common inputs, whereas the systems output is represented by the sum of all the neurons, as in conventional array-based SR systems.

Our key proposal here is that each neuron accepts temporal noise generated by the neuron's neighbors, i.e., the sum of asynchronous activities among the neighbors are considered as noise. To sustain the noise (neuron's activities), we introduced unidirectional coupling between the neurons, which results in one-way excitable wave propagation, avoiding extinction of excitable waves due to bidirectional wave collision.

Through extensive numerical simulations, we found that i) excitable waves propagated on the network in one way, and neuron's activities were sustained without the subthreshold input, ii) when the subthreshold input magnitude was increased, with the help of the sustained excitable waves (noise), neurons were able to resonate with the input stochastically, which represented SR without conventional (external) noise sources, and iii) the number of connected neighbors $(n)$, which characterized the amount of internal Gaussian noise, was the most important parameter as expected, and $n$ must be in a range of $[4,9]$ with our simulation setups, to ensure strong correlation between the input and the output.

The frequency response of the network has then be studied. Four input frequency domains have been identified, which quantitatively distinguish from their correlation values. The network only correctly operates in the two lower-frequency domains. Henceforth, a method enabling improving the frequency response which is based on optimizing the time constant has been proposed.

The proposed network demonstrates SR based on internally (or intrinsically) generated noise, which would significantly relax design constraints of hardware implementations. Exploiting noise in threshold systems as described in this paper may be used to improving the capability of audio or image sensors subthreshold signal detection. Moreover, the new SR scheme which utilizes internal noise in bistable systems may be considered a possible candidate for increasing memory or data link transmission reliability under aggressively noisy environments.

\section{Acknowledgments}

This study was supported by the JSPS Grants-in-Aid for JSPS Fellows, and a Grant-in-Aid for Scientific Research on Innovative Areas [2511001503] from the Ministry of Education, Culture, Sports, Science and Technology (MEXT) of Japan.

\section{References}

[1] J.K. Douglass, L. Wilkens, E. Pantazelou, and F. Moss, "Noise enhancement of information transfer in crayfish mechanoreceptors by stochastic resonance," Nature, vol. 365, no. 23, pp. 337340, 1993.

[2] M.C.W. van Rossum, B.J. O'Brien, and R.G. Smith, "Effects of noise on the spike timing precision of retinal ganglion cells," J. Neurophysiol., vol. 89, no. 5, pp. 2406-2419, 2003.

[3] B.C. Kim, J. Jeon, and H. Shin, "Temporal noise analysis and reduction method in CMOS image sensor readout circuit," IEEE Trans. Electron Devices, vol. 56, no. 11, pp. 2489-2495, 2009.

[4] C. Enz and G. Temes, "Circuit techniques for reducing the effect of op-amp imperfections: autozeroing, correlated double sampling and chopper stabilization," Proc. IEEE, vol. 84, no. 11, pp. 1584-1614, 1996.

[5] F. Peper, J. Lee, and L.B. Kish, "The exploitation of noise in computation," Proc. Int. Symp. Intelligent Signal Processing and Communication Systems, pp. 351-354, 2009. 
[6] T. Oya, "Thermal-noise-exploiting operations of single-electron majority logic circuits with conventional clock signals," IEEE Trans. Nanotechnology, vol. 11, no. 1, pp. 134-138, 2012.

[7] L.G.-Carabarin, T. Asai, and M. Motomura, "Impact of noise on spike transmission through serially-connected electrical FitzHugh-Nagumo circuits with subthreshold and suprathreshold interconductances," J. Signal Processing, vol. 16, no. 6, pp. 503-509, 2012.

[8] L.G.-Carabarin, T. Asai, and M. Motomura, "Application of nonlinear systems for designing low-power logic gates based on stochastic resonance," NOLTA, vol. 5, no. 4, pp. 445-455, 2014.

[9] L.G.-Carabarin, T. Asai, and M. Motomura, "Low-power asynchronous digital pipeline based on mismatch-tolerant logic gates," IEICE ELEX, vol. 11, no. 15, pp. 20140632/1-9, 2014.

[10] J.A. Hertz, A.S. Krogh, and R.G. Palmer, Introduction to the Theory of Neural Computation, Perseus Publishing, Massachusetts, 1991.

[11] J.J. Collins, C.C. Chow, and T.T. Imhoff, "Stochastic resonance without tuning," Nature, vol. 376, no. 20, pp. 236-238, 1995.

[12] I. Tokuda, C.E. Han, K. Aihara, M. Kawato, and N. Schweighofer, "The role of chaotic resonance in cerebellar learning," Neural Netw., vol. 23, no. 7, pp. 836-842, 2010.

[13] N. Schweighofer, K. Doya, H. Fukai, J.V. Chiron, T. Furukawa, and M. Kawato, "Chaos may enhance information transmission in the inferior olive," Proc. Natl. Acad. Sci., vol. 101, no. 13, pp. 4655-4660, 2004.

[14] K. Ishimura, T. Asai, and M. Motomura, "Chaotic resonance in forced Chua's oscillators," J. Signal Processing, vol. 17, no. 6, pp. 231-238, 2013.

[15] G.M. Tovar, T. Asai, and Y. Amemiya, "Array-enhanced stochastic resonance in a network of noisy neuromorphic circuits," Neural Information Processing: Theory and Algorithms, Lecture Notes in Computer Science, vol. 6443, pp. 188-195, Springer, New York, 2010.

[16] G. Nicolis, C. Nicolis, and D. McKernan, "Stochastic resonance in chaotic dynamics," J. Stat. Phys., vol. 70, nos. 1-2, pp. 125-139, 1993.

[17] V.S. Anishchenko, A.B. Neiman, and M.A. Safanova, "Stochastic resonance in chaotic systems," J. Stat. Phys., vol. 70, nos. 1-2, pp. 183-196, 1993.

[18] A. Crisanti, M. Falcioni, G. Paladin, and A. Vulpiani, "Stochastic resonance in deterministic chaotic systems," J. Phys. A: Math, vol. 27, no. 17, pp. 597-603, 1994.

[19] H. Nishimura, N. Katada, and K. Aihara, "Coherent response in a chaotic neural network," Neural Processing Lett., vol. 12, no. 1, pp. 49-58, 2000.

[20] J.D. Murray, Mathematical Biology I: An Introduction (3rd Ed.), Chap. 7, p. 239, Springer, New York, 2002.

[21] J.J. Collins, C.C. Chow, A.C. Capela, and T.T. Imhoff, "Aperiodic stochastic resonance," Phys. Rev. E, vol. 54, no. 5, pp. 5575-5584, 1996. 